\title{
Multiple Vascular Disruption Syndrome
}

National Cancer Institute

\section{Source}

National Cancer Institute. Multiple Vascular Disruption Syndrome. NCI Thesaurus. Code C129869.

A rare syndrome that refers to a constellation of anomalies resulting from multiple vascular disruption. 